\title{
FINITE AND COUNTABLE FAMILIES OF ALGEBRAS OF SETS
}

\author{
L.Š. GRINBlat
}

Abstract. Let $\left\{\mathcal{A}_{\lambda}\right\}_{\lambda \in \Lambda}$ be a family of algebras of sets defined on a set $X, 0<\#(\Lambda) \leq$ $\aleph_{0}$, and $\mathcal{A}_{\lambda} \neq \mathcal{P}(X)$ for each $\lambda \in \Lambda$. We assume that $\mathcal{A}_{\lambda}$ are $\sigma$-algebras if $\#(\Lambda)=\aleph_{0}$. We obtained the necessary and sufficient conditions for which $\bigcup_{\lambda \in \Lambda} \mathcal{A}_{\lambda}=\mathcal{P}(X)$. In the formulation of these conditions we use $\omega$-saturated algebras and finite sequences of ultrafilters on $X$.

\section{The formulation of results}

1.1. The object of our present investigation is algebras of sets. The present article is a further development of the theory formulated in [Gr1],[Gr2],[Gr3], [Gr4], [Gr5]. The results of other authors from $[\mathrm{E}],[\mathrm{S}],[\mathrm{G}-\mathrm{S}],[\mathrm{W}]$ bear a relation to the subject of our research.

Definition. By an algebra on a set $X$ we mean a non-empty system of subsets $X$ with the following properties: (1) if $M \in \mathcal{A}$, then $X \backslash M \in \mathcal{A}$; (2) if $M_{1}, M_{2} \in \mathcal{A}$, then $M_{1} \cup M_{2} \in \mathcal{A}$.

1.2. Some notations and terms. All algebras and measures are considered on some abstract set $X$. As usual, $\mathcal{P}(M)$ denotes the set of all subsets of the set $M$. When it is clear from the context, we will not state explicitly that a set belongs to $\mathcal{P}(X)$. The symbol \#(M) denotes the cardinality of the set $M$. The set $M$ is called countable if $\#(M)=\aleph_{0}$. We assume that $\#(X) \geq \aleph_{0}$. We denote the set of natural numbers by $\mathbb{N}^{+}$. If $n \in \mathbb{N}^{+}$, then we define

$$
\mathbb{N}_{n}=\left\{k \in \mathbb{N}^{+} \mid k \leq n\right\} .
$$

As usual, an algebra $\mathcal{A}$ is called a $\sigma$-algebra, if for any countable sequence $M_{1}, \ldots, M_{k}$, $\ldots \in \mathcal{A}$, we have that $\mathcal{A} \ni \bigcup_{k=1}^{\infty} M_{k}$. We will consider ultrafilters on $X$. Each ultrafilter is a point $\beta X$ and vice-versa - each point $\beta X$ is an ultrafilter on $X$. (Here, as usual, $\beta X$ is the Stone-Čech compactification of $X$ with discrete topology).

1.3. Example. There exists a finite sequence of algebras $\mathcal{A}_{1}, \ldots, \mathcal{A}_{\nu}$, where $\nu$ is an odd number $\geq 3$, and $\mathcal{A}_{i} \neq \mathcal{P}(X)$ for each $i \in \mathbb{N}_{\nu}$, such that $\bigcup_{i=1}^{\nu} \mathcal{A}_{i}=\mathcal{P}(X)$.

2000 Mathematics Subject Classification. Primary 03E05, Secondary 54D35.

Key words and phrases. Algebra of sets, $\sigma$-algebra, ultrafilter.

Received by the editors February 24, 2010. 
Construction. Consider pairwise distinct ultrafilters $a_{1}, \ldots, a_{\nu}$. Let $a_{\nu+1}=a_{1}$. For any $i \in \mathbb{N}_{\nu}$ we construct the algebra

$$
\mathcal{A}_{i}=\left\{Q \in \mathcal{P}(X) \mid \text { either } \quad \mathrm{Q} \in a_{i}, a_{i+1} \text { or } Q \notin a_{i}, a_{i+1}\right\} .
$$

We shall see that there is a close connection between this simple example and results which we want to prove.

1.4 Definition. We say that a pair $\{\mathfrak{A}, A\}$, where $\mathfrak{A}$ is a family of algebras and $A$ is a set of ultrafilters, forms a cycle, if the following conditions hold:

1) $\mathfrak{A}$ is a finite sequence of algebras $\mathcal{A}_{1}, \ldots, \mathcal{A}_{\nu}$ and $\nu$ is an odd number $\geq 3$;

2) $A$ is a set of pairwise distinct ultrafilters $a_{1}, \ldots, a_{\nu}$ (let $a_{\nu+1}=a_{1}$ );

3) if $i \in \mathbb{N}_{\nu}, Q \in a_{i}$ and $Q \notin a_{i+1}$, then $Q \notin \mathcal{A}_{i}$.

1.5. Definition. Let $\left\{\mathcal{A}_{\lambda}\right\}_{\lambda \in \Lambda}$ be a family of algebras. We say that this family has a cycle if there exists a pair $\{\mathfrak{A}, A\}$ forming the cycle; $\mathfrak{A}=\left\{\mathcal{A}_{\lambda_{1}}, \ldots, \mathcal{A}_{\lambda_{n}}\right\}$, and $\lambda_{1}, \ldots, \lambda_{n}$ are pairwise distinct indices from $\Lambda$.

1.6. Definition. Let $\left\{\mathcal{A}_{\lambda}\right\}_{\lambda \in \Lambda}$ be a family of algebras having a countable sequence cycles $\left\{\mathfrak{A}_{1}, A_{1}\right\}, \ldots,\left\{\mathfrak{A}_{k}, A_{k}\right\}, \ldots$. We say that this sequence is a countable splitting sequence if for each $n \in \mathbb{N}^{+}$the following conditions hold:

$$
\text { (1) } \mathfrak{A}_{n+1} \backslash \bigcup_{k=1}^{n} \mathfrak{A}_{k} \neq \emptyset \quad ; \quad \text { (2) } A_{n+1} \backslash \bigcup_{k=1}^{n} A_{k} \neq \emptyset .
$$

1.7. The next definition was introduced by other authors long before the publication of $[\mathrm{Gr} 1]$.

Definition. An algebra is said to be $\omega$-saturated if there does not exist an infinite number of pairwise disjoint sets not belonging to it.

Later on it becomes clear that an algebra $\mathcal{A}$ is $\omega$-saturated if and only if there exists a finite set of ultrafilters $G$ such that if $M$ belongs to all ultrafilters from $G$, then $M \in \mathcal{A}$.

1.8. Definition. A family of algebras is said to be strongly cyclical, if it has a cycle containing only $\omega$-saturated algebras.

1.9. Definition. A cycle $\{\mathfrak{A}, A\}$, where $\mathfrak{A}=\left\{\mathcal{A}_{1}, \ldots, \mathcal{A}_{\nu}\right\}$, is said to be a $\omega$-cycle, if each pair $\left(\mathcal{A}_{1}, \mathcal{A}_{2}\right),\left(\mathcal{A}_{2}, \mathcal{A}_{3}\right), \ldots,\left(\mathcal{A}_{\nu-1}, \mathcal{A}_{\nu}\right),\left(\mathcal{A}_{\nu}, \mathcal{A}_{1}\right)$ contains an $\omega$-saturated algebra.

It is clear that the majority algebras from $\mathfrak{A}$ are $\omega$-saturated. If algebras from $\mathfrak{A}$ are $\sigma$-algebras, then all ultrafilters from $A$ are irregular (see Sections 2.4, 2.11).

1.10. In this and in the following section we present theorems about necessary conditions for our results.

Theorem. Let $\mathfrak{A}=\left\{\mathcal{A}_{k}\right\}_{k \in \mathbb{N}_{n}}$ be a family of algebras, $\mathcal{A}_{k} \neq \mathcal{P}(X)$ for each $k \in \mathbb{N}_{n}$ and $\bigcup_{k=1}^{n} \mathcal{A}_{k}=\mathcal{P}(X)$. Then $\mathfrak{A}$ is strongly cyclical.

1.11. Theorem. Let $\mathfrak{A}=\left\{\mathcal{A}_{k}\right\}_{k \in \mathbb{N}^{+}}$be a family of $\sigma$-algebras, $\mathcal{A}_{k} \neq \mathcal{P}(X)$ for each $k \in \mathbb{N}^{+}$and $\bigcup_{k=1}^{\infty} \mathcal{A}_{k}=\mathcal{P}(X)$. Then, if $\mathfrak{A}$ is not strongly cyclical, it has countable splitting sequence of $\omega$-cycles. 
1.12. Remark. Using the notation of absolute introduced by Gleason in [Gl] we can construct the family of algebras $\left\{\mathcal{B}_{k}\right\}_{k \in \mathbb{N}^{+}}$possessing the following properties: each algebra is not $\omega$-saturated, not $\sigma$-algebra and $\bigcup_{k=1}^{\infty} \mathcal{B}_{k}=\mathcal{P}(X)$ (see [Gr2], Chapter $5)$. Therefore in Theorem 1.11 we consider a family of $\sigma$-algebras.

1.13. Definition. We say that an algebra $\mathcal{B}$ is the extension of an algebra $\mathcal{A}$ if $\mathcal{B} \supset \mathcal{A}$, and $\mathcal{B} \neq \mathcal{P}(X)$ if $\mathcal{A} \neq \mathcal{P}(X)$. We say that a family of algebras $\left\{\mathcal{B}_{\lambda}\right\}_{\lambda \in \Lambda}$ is the extension of a family of algebras $\left\{\mathcal{A}_{\lambda}\right\}_{\lambda \in \Lambda}$ if $\mathcal{B}_{\lambda}$ is the extension of $\mathcal{A}_{\lambda}$ for each $\lambda \in \Lambda$. We say that an algebra $\mathcal{B}$ is the $\sigma$-extension of an algebra $\mathcal{A}$ if $\mathcal{B}$ is the extension of $\mathcal{A}$ and $\mathcal{B}$ is a $\sigma$-algebra. Accordingly, we say that a family of algebras $\left\{\mathcal{B}_{\lambda}\right\}_{\lambda \in \Lambda}$ is the $\sigma$-extension of a family of algebras $\left\{\mathcal{A}_{\lambda}\right\}_{\lambda \in \Lambda}$.

1.14. In this and in the following section we present theorems about sufficient conditions for our results.

Theorem. Let $\mathfrak{A}=\left\{\mathcal{A}_{k}\right\}_{k \in \mathbb{N}_{n}}$ be a family of algebras and $\bigcup_{k=1}^{n} \mathcal{A}_{k} \neq \mathcal{P}(X)$. Then there exists an extension $\mathfrak{B}$ of $\mathfrak{A}$ such that $\mathfrak{B}$ has no cycle.

1.15. Theorem. Let $\mathfrak{A}=\left\{\mathcal{A}_{k}\right\}_{k \in \mathbb{N}^{+}}$be a family of $\sigma$-algebras and $\bigcup_{k=1}^{\infty} \mathcal{A}_{k} \neq \mathcal{P}(X)$. Then there exists a $\sigma$-extension $\mathfrak{B}$ of $\mathfrak{A}$ such that $\mathfrak{B}$ has no cycle.

1.16. Using Theorems 1.10 and 1.14 we can formulate the main result about finite families of algebras.

Theorem. Let $\mathfrak{A}=\left\{\mathcal{A}_{k}\right\}_{k \in \mathbb{N}_{n}}$ be a family of algebras, and $\mathcal{A}_{k} \neq \mathcal{P}(X)$ for each $k \in \mathbb{N}_{n}$. Then we have: $\bigcup_{k=1}^{n} \mathcal{A}_{k}=\mathcal{P}(X)$ if and only if any extension of $\mathfrak{A}$ is strongly cyclical.

1.17. Using Theorems 1.11 and 1.15 we can formulate the main result about countable families of $\sigma$-algebras.

Theorem. Let $\mathfrak{A}=\left\{\mathcal{A}_{k}\right\}_{k \in \mathbb{N}^{+}}$be a family of $\sigma$-algebras, and $\mathcal{A}_{k} \neq \mathcal{P}(X)$ for each $k \in \mathbb{N}^{+}$. Then we have: $\bigcup_{k=1}^{\infty} \mathcal{A}_{k}=\mathcal{P}(X)$ if and only if for any $\sigma$-extension $\mathfrak{B}$ of $\mathfrak{A}$ one of the following two conditions holds:

(1) $\mathfrak{B}$ is strongly cyclical;

(2) $\mathfrak{B}$ is not strongly cyclical, and $\mathfrak{B}$ has countable splitting sequence of $\omega$-cycles.

\section{On necessary conditions}

2.1. In the first 13 sections of this chapter we present information from [Gr1] and [Gr2]. In this section we provide the important statement used in our previous publications. Consider an algebra $\mathcal{A}$. Two ultrafilters $a \neq b$ are said to be $\mathcal{A}$-equivalent if for any $M \in \mathcal{A}$ we have that $M \in a, b$ or $M \notin a, b$. The $\mathcal{A}$-equivalent relation is symmetric and transitive. If $a, b$ are $\mathcal{A}$-equivalent ultrafilters then we say that $a$ has an $\mathcal{A}$-equivalent ultrafilter $b$, or a is $\mathcal{A}$-equivalent to $b$. It is clear that if $\mathcal{A}=\mathcal{P}(X)$, then $\mathcal{A}$-equivalent ultrafilters do not exist. It is not complicated to prove that $Q \notin \mathcal{A}$ if and only if there exist $\mathcal{A}$-equivalent ultrafilters $a, b$ such that $Q \in a, X \backslash Q \in b$. 
Main Statement. Let $\left\{\mathcal{A}_{k}\right\}_{\lambda \in \Lambda}$ be a family of algebras. Then $\bigcup_{\lambda \in \Lambda} \mathcal{A}_{\lambda} \neq \mathcal{P}(X)$ if and only if there exist closed disjoint sets $S, T \subset \beta X$ and for each $\lambda \in \Lambda$ the following is true: there exist $\mathcal{A}_{\lambda}$-equivalent ultrafilters $s_{\lambda}, t_{\lambda}$ such that $s_{\lambda} \in S$ and $t_{\lambda} \in T$.

2.2. Consider a family of algebras $\left\{\mathcal{A}_{k}\right\}_{k \in \mathbb{N}_{n}}$. By the Main Statement $2.1 \bigcup_{k=1}^{n} \mathcal{A}_{k} \neq$ $\mathcal{P}(X)$ if and only if there exist two disjoint sets $S_{n}, T_{n} \subset \beta X$ such that:

- $S_{n}=\left\{s_{1}, \ldots, s_{i}, \ldots, s_{n}\right\}$, where in general it is possible that $s_{i}=s_{j}(i \neq j)$;

- $T_{n}=\left\{t_{1}, \ldots, t_{i}, \ldots, t_{n}\right\}$, where in general it is possible that $t_{k}=t_{l}(k \neq l)$;

- $s_{i}, t_{i}$ are $\mathcal{A}_{i}$ - equivalent ultrafilters for each $i \in \mathbb{N}_{n}$.

2.3. Definition. Consider an algebra $\mathcal{A}$; the set

$$
\{a \in \beta X \mid a \text { has } \mathcal{A} \text {-equivalent ultrafilter }\}
$$

is called the kernel of the algebra $\mathcal{A}$ and is denoted by $\operatorname{ker} \mathcal{A}$.

If an algebra $\mathcal{A}=\mathcal{P}(X)$, then $\operatorname{ker} \mathcal{A}=\emptyset$. If an algebra $\mathcal{A} \neq \mathcal{P}(X)$, then $\#(\operatorname{ker} \mathcal{A}) \geq$ 2. An algebra $\mathcal{A}$ is $\omega$-saturated if and only $\#(\operatorname{ker} \mathcal{A})<\aleph_{0}$; the minimal set $G$ from Section 1.7 is $\operatorname{ker} \mathcal{A}$.

2.4. Notation: if $M \subset \beta X$, then by $\bar{M}$ we denote the close $M$ in $\beta X$.

Definition. A point $a \in \beta X$ is said to be irregular if for any countable sequence of sets $M_{1}, \ldots, M_{k}, \ldots \subset \beta X$ such that $a \notin \bar{M}_{k}$ for all $k$, we have that $a \notin \bar{\bigcup}_{k}$. A point of $\beta X$ is said to be regular if it is not irregular.

Since a point of $\beta X$ is an ultrafilter on $X$ and vice versa, an ultrafilter on $X$ is a point of $\beta X$, we will use the notion of the irregular (regular) ultrafilter along with the notion of the irregular (regular) point. All points of $X$ are irregular. Just a few words about irregular points in $\beta X \backslash X$. The superposition of the absence of irregular points in $\beta X \backslash X$ at an arbitrary cardinality of $X$ is consistent, as is known, with the usual axioms of set theory. However, it is possible to construct models with irregular points in $\beta X \backslash X$ when the cardinality of $X$ is "very large".

2.5. Definition. An algebra $\mathcal{A}$ is said to be simple, if there exists $Z \subset \beta X$ such that:

(1) $0<\#(Z) \leq \aleph_{0}$

(2) all points of $Z$ are irregular;

(3) $\operatorname{ker} \mathcal{A} \subset \bar{Z}$.

2.6. Remark. By virtue of Definition 2.5 the algebra $\mathcal{P}(X)$ is simple.

2.7. The following theorem was proved in [Gr1], Chapter 5 .

Theorem. Let $\left\{\mathcal{A}_{k}\right\}_{k \in \mathbb{N}^{+}}$be a family of $\sigma$-algebras, and let

$$
\bigcup\left\{\mathcal{A}_{k} \mid \mathcal{A}_{k} \text { is a simple algebra }\right\} \neq \mathcal{P}(X) .
$$

Then $\bigcup_{k=1}^{\infty} \mathcal{A}_{k} \neq \mathcal{P}(X)$.

2.8. Remark. The Gitik-Shelah theorem [G-S] is essentially used in the proof of Theorem 2.7. We now formulate the deep theorem of Gitik-Shelah. 
Given countable sequence of $\sigma$-additive measures $\mu_{1}, \ldots, \mu_{k}, \ldots$ on $X, \#(X)=2^{\aleph_{0}}$, and such that for each $k \in \mathbb{N}^{+}$the following conditions hold:

(1) $\mu_{k}(\{x\})=0$ if $x \in X$;

(2) $\mu_{k}(X)=1$

(3) if $M$ is $\mu_{k}$ - measurable set, then either $\mu_{k}(M)=0$ or $\mu_{k}(M)=1$.

Then there exists a set that is nonmeasurable with respect to each of these measures.

2.9. Definition. The set

$$
\{a \in \operatorname{ker} \mathcal{A} \mid a \text { is an irregular point }\}
$$

is called the spectrum of an algebra $\mathcal{A}$ and is denoted by $\operatorname{sp} \mathcal{A}$.

2.10. The proof of the lemma below can be found in [Gr2], Chapter 7 .

Lemma. If $\mathcal{A}$ is a simple $\sigma$-algebra, then $\operatorname{ker} \mathcal{A} \subset \overline{s p \mathcal{A}}$.

2.11. Remark. If an $\omega$-saturated algebra $\mathcal{A}$ is a $\sigma$-algebra, then $\mathcal{A}$ is simple and $\operatorname{ker} \mathcal{A}=s p \mathcal{A}$. It is clear that if all algebras of an $\omega$-cycle are $\sigma$-algebras, then all ultrafilters of this cycle are irregular.

2.12. Definition. A simple but not $\omega$-saturated algebra is called strictly simple.

2.13. Let us mention the next simple lemma.

Lemma. Let $\mathcal{A}$ be a strictly simple $\sigma$-algebra, and $M$ a finite set such that $M \subset s p \mathcal{A}$. Then there exist $\mathcal{A}$-equivalent ultrafilters $a, b$ such that $a, b \in \operatorname{sp} \mathcal{A} \backslash M$.

2.14. In this and the following section we prove Theorem 1.10 .

Theorem. Let $\mathfrak{A}=\left\{\mathcal{A}_{k}\right\}_{k \in \mathbb{N}_{n}}$ be a family of algebras, $\mathcal{A}_{k} \neq \mathcal{P}(X)$ for each $k \in \mathbb{N}_{n}$, and $\bigcup_{k=1}^{n} \mathcal{A}_{k}=\mathcal{P}(X)$. Then $\mathfrak{A}$ has a cycle.

Proof. Let $m$ be the maximum natural number such that $\bigcup_{k=1}^{m} \mathcal{A}_{k} \neq \mathcal{P}(X)$ and $\bigcup_{k=1}^{m+1} \mathcal{A}_{k}=\mathcal{P}(X)$. It is clear that $m \geq 2$ and for algebras $\mathcal{A}_{1}, \ldots, \mathcal{A}_{m}$ there exist the corresponding sets $S_{m}, T_{m}$ (see Section 2.2);

$$
\begin{aligned}
S_{m} & =\left\{s_{1}, \ldots, s_{m}\right\}, \\
T_{m} & =\left\{t_{1}, \ldots, t_{m}\right\}
\end{aligned}
$$

etc. Let $a, b$ be $\mathcal{A}_{m+1}$-equivalent ultrafilters. It is clear that either $a, b \in S_{m}$ or $a, b \in$ $T_{m}$. (If we suppose the contrary, then it is possible to construct the corresponding sets $S_{m+1}, T_{m+1}$, and $\left.\bigcup_{k=1}^{m+1} \mathcal{A}_{k} \neq \mathcal{P}(X)\right)$. Let $a, b \in S_{m}$. Setting

$$
\begin{aligned}
S^{(1)} & =\{a\}, \\
T^{(1)} & =\left\{t_{k} \in T_{m} \mid s_{k}=a\right\}, \\
S^{(2)} & =\left\{s_{k} \in S_{m} \mid t_{k} \in T^{(1)}\right\}
\end{aligned}
$$

etc. It is clear that $S^{(1)} \subset S^{(2)} \subset \ldots$, and $T^{(1)} \subset T^{(2)} \subset \ldots$. Consider two cases:

(1) there exists minimal $\nu$ such that $b \in S^{(\nu)}$;

(2) such $\nu$ does not exist. 
Suppose that (2) holds. We continue this process to construct sets $S^{(i)}, T^{(j)}$, until it terminates. Let $S_{*}$ denote the last set $S^{(i)}$. Let $T_{*}$ denote the last set $T^{(j)}$. Each $s_{k} \in$ $S_{*}$ we denote by $t_{k}$, and each set $t_{k} \in T_{*}$ we denote by $s_{k}$. Put $s_{m+1}=b, t_{m+1}=a$. Other $s_{k}$ and $t_{k}$ do not change the notations. We constructed the corresponding sets $S_{m+1}, T_{m+1}$, and therefore $\bigcup_{k=1}^{m+1} \mathcal{A}_{k} \neq \mathcal{P}(X)$. We arrive at the contradiction. So, the case (1) is true. There exist pairwise distinct numbers

$$
k_{1}, k_{2}, \ldots, k_{2 \nu-2} \in \mathbb{N}_{m}
$$

and pairwise distinct ultrafilters

$$
a=s_{k_{1}}, s_{k_{3}} \in S^{(2)}, \ldots, s_{2 \nu-3} \in S^{(\nu-1)}, b=s_{2 \nu-2} \in S^{(\nu)},
$$

and pairwise distinct ultrafilters

$$
t_{k_{2}} \in T^{(1)}, \ldots, t_{2 \nu-2} \in T^{(\nu-1)}
$$

such that

$$
\begin{aligned}
& s_{k_{1}}, t_{k_{2}} \text { are } \mathcal{A}_{k_{1}} \text {-equivalent ultrafilters }\left(t_{k_{1}}=t_{k_{2}}\right) \text {, } \\
& t_{k_{2}}, s_{k_{3}} \text { are } \mathcal{A}_{k_{2}} \text {-equivalent ultrafilters }\left(s_{k_{2}}=s_{k_{3}}\right) \text {, } \\
& s_{k_{2 \nu-3}}, t_{k_{2 \nu-2}} \text { are } \mathcal{A}_{2 \nu-3} \text {-equivalent ultrafilters }\left(t_{k_{2 \nu-3}}=t_{k_{2 \nu-2}}\right) \text {, } \\
& t_{k_{2 \nu-2}}, s_{k_{2 \nu-2}} \text { are } \mathcal{A}_{2 \nu-2} \text {-equivalent ultrafilters. }
\end{aligned}
$$

In addition, $a$ and $b$ and $\mathcal{A}_{m+1}$-equivalent ultrafilters. We constructed the cycle.

2.15. Remark. Returning to Theorem 2.14 , let us divide the family $\mathfrak{A}$ into two subfamilies:

$$
\mathfrak{C}=\left\{\mathcal{C}_{1}, \ldots, \mathcal{C}_{n_{1}}\right\}
$$

and

$$
\mathfrak{D}=\left\{\mathcal{D}_{1}, \ldots, \mathcal{D}_{n_{2}}\right\}
$$

$\mathfrak{C}$ includes only $\omega$-saturated algebras. $\mathfrak{D}$ does not include $\omega$-saturated algebras. Suppose that $\mathfrak{D} \neq \emptyset$. Let

$$
L=\bigcup_{k=1}^{n_{1}} \operatorname{ker} \mathcal{C}_{k}
$$

We know that $\#(L)<\aleph_{0}$, and $\#\left(\operatorname{ker} \mathcal{D}_{k}\right) \geq \aleph_{0}$ for each $k \in \mathbb{N}_{n_{2}} .{ }^{1}$ It is clear that there exist pairwise distinct ultrafilters

$$
a_{1}, b_{1}, \ldots, a_{n_{2}}, b_{n_{2}}
$$

such that $a_{k}, b_{k}$ are $\mathcal{D}_{k}$ - equivalent ultrafilters for each $k \in \mathbb{N}_{n_{2}}$, and

$$
L \cap\left\{a_{1}, b_{1}, \ldots, a_{n_{2}}, b_{n_{2}}\right\}=\emptyset .
$$

Therefore we have: $\mathfrak{C} \neq \emptyset$ and $\bigcup_{k=1}^{n_{1}} \mathcal{C}_{k}=\mathcal{P}(X)$. By Theorem 2.14 we have: $\mathfrak{A}$ is strongly cyclical.

\footnotetext{
${ }^{1}$ In [Gr2], Chapter 3 it was proved that there does not exist an algebra $\mathcal{A}$ such that
}

$$
\aleph_{0} \leq \#(\operatorname{ker} \mathcal{A})<2^{2^{\aleph_{0}}} \text {. }
$$




\subsection{The proof of Theorem 1.11. Let}

$$
\mathfrak{A}^{\prime}=\left\{\mathcal{A}_{k} \in \mathfrak{A} \mid \mathcal{A}_{k} \quad \text { is a simple algebra }\right\} .
$$

By Theorem 2.7 we have $\bigcup \mathfrak{A}^{\prime}=\mathcal{P}(X)$. Suppose that $\#\left(\mathfrak{A}^{\prime}\right)<\aleph_{0}$. We know that the family $\mathfrak{A}^{\prime}$ is not strongly cyclical. Therefore by the considerations of Remark 2.15 we have: $\bigcup \mathfrak{A}^{\prime} \neq \mathcal{P}(X)$. By this contradiction we have $\#\left(\mathfrak{A}^{\prime}\right)=\aleph_{0}$. Put

$$
\mathfrak{A}^{\prime}=\left\{\mathcal{A}_{k}^{\prime}\right\}_{k \in \mathbb{N}^{+}}, Z=\bigcup_{k=1}^{\infty} s p \mathcal{A}_{k}^{\prime} .
$$

Let us divide $\mathfrak{A}^{\prime}$ into two subsequences:

$$
\text { (1) } \mathcal{C}_{1}, \ldots, \mathcal{C}_{k}, \ldots
$$

and

(2) $\mathcal{D}_{1}, \ldots, \mathcal{D}_{k}, \ldots$.

The first subsequence includes all $\omega$-saturated algebras. The second subsequence includes all strictly simple algebras. We recall that $\operatorname{ker} \mathcal{C}_{k} \subset Z$. Let $\mathcal{C}_{1}, \ldots, \mathcal{C}_{n}$ be algebras where $n \in \mathbb{N}^{+}$. We know that $\bigcup_{k=1}^{n} \mathcal{C}_{k} \neq \mathcal{P}(X)$ and there exist corresponding sets of ultrafilters $S_{n}, T_{n} \subset Z$. By Lemma 2.13 we have: if (1) includes only a finite number of algebras or if (1) is empty, then $\bigcup_{k=1}^{\infty} \mathcal{A}_{k}^{\prime} \neq \mathcal{P}(X)$. Therefore (1) has an infinite number of algebras. Since $\#\left(\operatorname{ker} \mathcal{C}_{k}\right)<\aleph_{0}$, we can construct disjoint sets $S, T \subset Z$ such that the following is true: for each $k \in \mathbb{N}^{+}$there exist $\mathcal{C}_{k}$-equivalent ultrafilters $\sigma_{k}, \tau_{k}$ such that $\sigma_{k} \in S, \tau_{k} \in T$. Since $S \cup T$ includes only irregular ultrafilters and $\#(S \cup T) \leq \aleph_{0}$, then $\bar{S} \cap \bar{T}=\emptyset$. By Main Statement 2.1 we have $\bigcup_{k=1}^{\infty} \mathcal{C}_{k} \neq \mathcal{P}(X)$. From this it follows that (2) is not empty. We assume, without any loss of generality, that (2) has an infinite number of algebras. By Lemma 2.13 we shall construct a countable sequence of pairwise distinct ultrafilters from $Z$ :

$$
a_{1}^{(1)}, b_{1}^{(1)}, a_{2}^{(1)}, b_{2}^{(1)}, \ldots, a_{k}^{(1)}, b_{k}^{(1)}, \ldots
$$

such that $a_{k}^{(1)}, b_{k}^{(1)}$ are $\mathcal{D}_{k}$-equivalent ultrafilters for each $k \in \mathbb{N}^{+}$. For each $k \in \mathbb{N}^{+}$ we consider the algebra $\mathcal{D}_{k}^{(1)}$ for which

$$
\operatorname{ker} \mathcal{D}_{k}^{(1)}=\left\{a_{k}^{(1)}, b_{n}^{(1)}\right\} .
$$

Consider the countable sequence of $\omega$-saturated algebras

$$
\mathcal{C}_{1}, \mathcal{D}_{1}^{(1)}, \mathcal{C}_{2}, \mathcal{D}_{2}^{(1)}, \ldots, \mathcal{C}_{k}, \mathcal{D}_{k}^{(1)}, \ldots
$$

If this sequence has no cycles, we arrive at the contradiction: $\bigcup_{k=1}^{\infty} \mathcal{A}_{k}^{\prime} \neq \mathcal{P}(X)$. Therefore this sequence of algebras has a cycle $\mathfrak{C}_{1}$. It is obvious that $\mathfrak{C}_{1}$ is the cycle of the countable sequence of algebras

$$
\mathcal{C}_{1}, \mathcal{D}_{1}, \mathcal{C}_{2}, \mathcal{D}_{2}, \ldots, \mathcal{C}_{k}, \mathcal{D}_{k}, \ldots
$$

Since the ultrafilters $a_{1}^{(1)}, b_{1}^{(1)}, \ldots, a_{k}^{(1)}, b_{k}^{(1)}, \ldots$ are pairwise distinct, then $\mathfrak{C}_{1}$ is $\omega$-cycle. It is clear that $\mathfrak{C}_{1}$ contains algebras of kind $\mathcal{D}_{k}$.

Consider a countable sequence of pairwise distinct ultrafilters from $Z$

$$
a_{1}^{(2)}, b_{1}^{(2)}, a_{2}^{(2)}, b_{2}^{(2)}, \ldots, a_{k}^{(2)}, b_{k}^{(2)}, \ldots .
$$


If $\mathcal{C}_{k} \in \mathfrak{C}_{1}$ then

$$
\operatorname{ker} \mathcal{C}_{k} \bigcap\left\{a_{1}^{(2)}, b_{1}^{(2)}, a_{2}^{(2)}, b_{2}^{(2)}, \ldots, a_{k}^{(2)}, b_{k}^{(2)}, \ldots\right\}=\emptyset .
$$

Moreover, $a_{k}^{(2)}, b_{k}^{(2)}$ are $\mathcal{D}_{k}$ - equivalent ultrafilters for each $k \in \mathbb{N}^{+}$. For each $k \in \mathbb{N}^{+}$ we consider the algebra $\mathcal{D}_{k}^{2}$ for which

$$
\operatorname{ker} \mathcal{D}_{k}^{(2)}=\left\{a_{k}^{(2)}, b_{k}^{(2)}\right\}
$$

Consider the countable sequence of $\omega$ - saturated algebras

$$
\mathcal{C}_{1}, \mathcal{D}_{1}^{(2)}, \mathcal{C}_{2}, \mathcal{D}_{2}^{(2)}, \ldots, \mathcal{C}_{k}, \mathcal{D}_{k}^{(2)}, \ldots
$$

This sequence has a cycle $\mathfrak{C}_{2}$. It is obvious that $\mathfrak{C}_{2}$ is the cycle of the countable sequence of algebras

$$
\mathcal{C}_{1}, \mathcal{D}_{1}, \mathcal{C}_{2}, \mathcal{D}_{2}, \ldots, \mathcal{C}_{k}, \mathcal{D}_{k}, \ldots
$$

It is clear that $\mathfrak{C}_{2}$ is $\omega$-cycle, and $\mathfrak{C}_{2}$ contains algebras of kind $\mathcal{D}_{k}$.

Consider a countable sequence of pairwise distinct ultrafilters from $Z$

$$
a_{1}^{(3)}, b_{1}^{(3)}, a_{2}^{(3)}, b_{2}^{(3)}, \ldots, a_{k}^{(3)}, b_{k}^{(3)}, \ldots .
$$

If $\mathcal{C}_{k}$ belongs either to $\mathfrak{C}_{1}$ or $\mathfrak{C}_{2}$, then

$$
\operatorname{ker} \mathcal{C}_{k} \bigcap\left\{a_{1}^{(3)}, b_{1}^{(3)}, a_{2}^{(3)}, b_{2}^{(3)}, \ldots, a_{k}^{(3)}, b_{k}^{(3)}, \ldots\right\}=\emptyset .
$$

Moreover, $a_{k}^{(3)}, b_{k}^{(3)}$ are $\mathcal{D}_{k}$-equivalent ultrafilters for each $k \in \mathbb{N}^{+}$etc. Continuing our process we shall construct a countable splitting sequence of $\omega$-cycles.

2.17. Now we turn to the necessary conditions of the main Theorem 1.17. In this theorem it is necessary to assume that $\mathcal{B}_{k}$ are $\sigma$-algebras.

Theorem. There exist families of $\sigma$-algebras $\mathfrak{A}=\left\{\mathcal{A}_{k}\right\}_{k \in \mathbb{N}^{+}}$, and the following is true:

I. $\bigcup_{k=1}^{\infty} \mathcal{A}_{k}=\mathcal{P}(X)$;

II. $\bigcup\left\{\mathcal{A}_{k} \mid \mathcal{A}_{k}\right.$ is $\omega$-saturated algebra $\} \neq \mathcal{P}(X)$.

We approve that for such a family $\mathfrak{A}$ there exists an extension $\mathfrak{B}$ which does not contain any cycle. ${ }^{2}$

Proof. It is clear that there exist families of $\sigma$-algebras $\mathfrak{A}=\left\{\mathcal{A}_{k}\right\}_{k \in \mathbb{N}^{+}}$with the conditions I and II. Let us divide $\mathfrak{A}$ into two subsequences:

$$
\text { (1) } \mathcal{C}_{1}, \ldots, \mathcal{C}_{k}, \ldots
$$

and

$$
\text { (2) } \mathcal{D}_{1}, \ldots, \mathcal{D}_{k}, \ldots \text {. }
$$

The first subsequence includes all $\omega$-saturated algebras. The second subsequence includes all other algebras. It is clear that (1) includes an infinite number of algebras and (2) is not empty. Suppose that (2) contains an infinite number of algebras. For each $k \in \mathbb{N}^{+}$we can take $\mathcal{C}_{k}$-equivalent ultrafilters $\sigma_{k}, \tau_{k}$ such that $S \cap T=\emptyset$, where $S=\bigcup\left\{\sigma_{k}\right\}, T=\bigcup\left\{\tau_{k}\right\}$. For each $k \in \mathbb{N}^{+}$we consider the algebra $\mathcal{C}_{k}^{*}$ with

$$
\operatorname{ker} \mathcal{C}_{k}^{*}=\left\{\sigma_{k}, \tau_{k}\right\}
$$

\footnotetext{
${ }^{2}$ It is obvious that $\mathfrak{B}$ is not a $\sigma$-extension of $\mathfrak{A}$.
} 
It is clear (see the footnote 1) that there exist pairwise distinct ultrafilters

$$
a_{1}, b_{1}, a_{2}, b_{2}, \ldots, a_{k}, b_{k}, \ldots
$$

such that $a_{k}, b_{k}$ are $\mathcal{D}_{k}$-equivalent ultrafilters, and

$$
(S \cup T) \cap\left\{a_{1}, b_{1}, \ldots, a_{k}, b_{k}, \ldots\right\}=\emptyset .
$$

For each ker $\in \mathbb{N}^{+}$we consider the algebra $\mathcal{D}_{k}^{*}$ with

$$
\operatorname{ker} \mathcal{D}_{k}^{*}=\left\{a_{k}, b_{k}\right\} \cdot^{3}
$$

We have the extension

$$
\mathfrak{B}=\left\{\mathcal{C}_{1}^{*}, \ldots, \mathcal{C}_{k}^{*}, \ldots, \mathcal{D}_{1}^{*}, \ldots, \mathcal{D}_{k}^{*}, \ldots\right\}
$$

of $\mathfrak{A}$. If $\mathfrak{B}$ has a cycle $\left\{\mathfrak{B}^{*}, B^{*}\right\}$ then

$$
\begin{aligned}
\mathfrak{B}^{*} & =\left\{\mathcal{C}_{k_{1}}^{*}, \ldots, \mathcal{C}_{k_{\nu}}^{*}\right\}, \\
B^{*} & =\left\{q_{k_{1}}, \ldots, q_{k_{\nu}}\right\} \subset S \cup T,
\end{aligned}
$$

$\nu$ is an odd number $\geq 3$ etc. If $q_{k_{1}} \in S$ then $q_{k_{1}}=\sigma_{k_{1}}, q_{k_{2}}=\tau_{k_{1}}=\tau_{k_{2}}, q_{k_{3}}=\sigma_{k_{2}}$. If $\nu>3$ then $\sigma_{k_{2}}=\sigma_{k_{3}}, q_{k_{4}}=\tau_{k_{3}}$ etc. It is clear that $q_{k_{\nu}}=\sigma_{k_{\nu-1}}$. However, $\sigma_{k_{1}}, \sigma_{k_{\nu-1}} \in S$, and therefore they are not $\mathcal{C}_{k_{\nu}}^{*}$-equivalent ultrafilters. We arrive at a contradiction; $\mathfrak{B}$ does not contain any cycle.

\section{On sufficient conditions}

3.1. The proof of Theorem 1.14. Consider the corresponding sets of ultrafilters $S_{n}, T_{n}$ (see Section 2.2). For each $k \in \mathbb{N}_{n}$ consider the algebra $\mathcal{B}_{k}$ with ker $\mathcal{B}_{k}=$ $\left\{s_{k}, t_{k}\right\}$. The family of algebras $\mathfrak{B}=\left\{\mathcal{B}_{k}\right\}_{k \in \mathbb{N}_{n}}$ has no cycle (see the end of the proof of Theorem 2.17).

3.2. We begin the preparation for the proof of Theorem 1.15 .

Definition. Let $\mathcal{A}$ be an algebra and $U \in \mathcal{P}(X)$. We denote by $\mathcal{A}_{U}$ the algebra $\{Q \in \mathcal{P}(X) \mid$ there exists $V \in \mathcal{A}$ such that $Q \cap U=V \cap U\}$.

It is clear that $\mathcal{A}_{U} \supset \mathcal{A}$. It is clear that if $\mathcal{A}$ is a $\sigma$-algebra, then $\mathcal{A}_{U}$ is a $\sigma$-algebra.

3.3. The proof of the next lemma is not difficult.

Lemma. We have $\mathcal{A}_{U} \neq \mathcal{P}(X)$ if and only if there exist $\mathcal{A}$-equivalent ultrafilters $a, b$ such that $U \in a, b$.

3.4. The proof of the next lemma is not difficult.

Lemma. Let $\mathcal{A}$ be a $\sigma$-algebra, $S$ and $T$ are disjoint sets of irregular ultrafilters, $\#(S \cup T)=\aleph_{0}$, and $s, t$ are $\mathcal{A}$-equivalent ultrafilters $s \in \bar{S}, t \in \bar{T}$. Then there exist $\mathcal{A}$-equivalent ultrafilters $s^{\prime}$ and $t^{\prime}$ such that $s^{\prime} \in S, t^{\prime} \in T$.

3.5. Lemma. Let $\left\{\mathcal{A}_{k}\right\}_{k \in \mathbb{N}^{+}}$be a family of simple $\sigma$-algebras and $\bigcup_{k=1}^{\infty} \mathcal{A}_{k} \neq \mathcal{P}(X)$. Then there exist disjoint sets of irregular ultrafilters $S$ and $T$ such that $\#(S \cup T) \leq \aleph_{0}$, and for each $k \in \mathbb{N}^{+}$there exist $\mathcal{A}_{k}$-equivalent ultrafilters $s_{k}, t_{k}$, where $s_{k} \in S, t_{k} \in T$.

\footnotetext{
${ }^{3}$ There exists, necessarily, a non $\sigma$-algebra between algebras $\mathcal{D}_{k}^{*}$.
} 
Proof. There exist disjoint sets $U$ and $V$ such that for each $k \in \mathbb{N}^{+}$there exist $\mathcal{A}_{k}$-equivalent ultrafilters $\sigma_{k}, \tau_{k}$ and $\sigma_{k} \ni U, \tau_{k} \ni V$. If $\sigma_{k}$ is a regular ultrafilter (it is possible if $\mathcal{A}_{k}$ is strictly simple algebra), then there exists a countable set of irregular points $S_{(k)} \subset \bar{U}$ and $\sigma_{k} \in \bar{S}_{(k)}$. By analogy, if $\tau_{k}$ is a regular ultrafilter then there exists a countable set of irregular points $T_{(k)} \subset \bar{V}$ and $\tau_{k} \in \bar{T}_{(k)}$. Therefore for each $k \in \mathbb{N}^{+}$there exist at most countable sets of irregular points $S_{(k)}, T_{(k)}$ such that

$$
\sigma_{k} \in \bar{S}_{(k)} \subset \bar{U}, \quad \tau_{k} \in \bar{T}_{(k)} \subset \bar{V} .
$$

By Lemma 3.4 for each $k \in \mathbb{N}^{+}$there exists $\mathcal{A}_{k}$-equivalent irregular ultrafilters $s_{k}$ and $t_{k}$ such that $s_{k} \ni U, t_{k} \ni V$. Put

$$
S=\left\{s_{k}\right\}_{k \in \mathbb{N}^{+}}, \quad T=\left\{t_{k}\right\}_{k \in \mathbb{N}^{+}} .
$$

3.6. The next theorem from [Gr2], Chapter 17 is a generalization of Theorem 2.7

Theorem. Let $\left\{\mathcal{A}_{k}\right\}_{k \in \mathbb{N}^{+}}$and $\left\{\mathcal{B}_{k}\right\}_{k \in \mathbb{N}^{+}}$be two families of $\sigma$-algebras. Let all the algebras $\mathcal{A}_{k}$ be simple, and along the algebras $\mathcal{B}_{k}$ let there be no simple algebras. Then there exist pairwise disjoint sets $W, U_{1}, \ldots, U_{k}, \ldots, V_{1}, \ldots, V_{k}, \ldots$ such that

(1) $\operatorname{ker} \mathcal{A}_{k} \subset \bar{W}$ for all $k \in \mathbb{N}^{+}$;

(2) if a set $Q$ contains one of the two sets $U_{k}, V_{k}$ and its intersection with the other set is empty, then $Q \notin \mathcal{B}_{k}$.

It is clear that in the proof of this theorem the Gitik-Shelah theorem is used (see Remark 2.8). It is clear that for each $k \in \mathbb{N}^{+}$there exists $\mathcal{B}_{k}$-equivalent ultrafilters $a_{k}, b_{k}$ such that $U_{k} \in a_{k}, V_{k} \in b_{k}$.

3.7. Proof of Theorem 1.15. We assume, without any loss of generality, that it is possible to divide the family $\mathfrak{A}$ into two subfamilies:

$$
\text { (1) }\left\{\mathcal{C}^{(k)}\right\}_{k \in \mathbb{N}^{+}}, \quad(2) \quad\left\{\mathcal{D}^{(k)}\right\}_{k \in \mathbb{N}^{+}} .
$$

The subfamily (1) includes all simple algebras. The subfamily (2) includes all other algebras. By Lemma 3.5 and Theorem 3.6 there exist disjoint sets of irregular ultrafilters $S, T$ and pairwise disjoint sets $U_{1}^{1}, U_{2}^{1}, U_{1}^{2}, U_{2}^{2}, \ldots, U_{1}^{k}, U_{2}^{k}, \ldots$ such that the following holds:

(1) $\#(S \cup T) \leq \aleph_{0}$;

(2) for each $k \in \mathbb{N}^{+}$there exist $\mathcal{C}^{(k)}$-equivalent ultrafilters $s_{k}, t_{k}$ such that $s_{k} \in$ $S, t_{k} \in T$

(3) $(\bar{S} \cup \bar{T}) \cap\left(\overline{U_{1}^{k}} \cup \overline{U_{2}^{k}}\right)=\emptyset$ for each $k \in \mathbb{N}^{+}$;

(4) for each $k \in \mathbb{N}^{+}$there exist $\mathcal{D}^{(k)}$-equivalent ultrafilters $a_{k}, b_{k}$ such that $U_{1}^{k} \in$ $a_{k}, U_{2}^{k} \in b_{k}$.

Put

$$
V_{k}=U_{1}^{k} \cup U_{2}^{k} .
$$

For each $k \in \mathbb{N}^{+}$we consider two $\sigma$ - algebras $\mathcal{B}_{k}^{\prime}, \mathcal{B}_{k}^{\prime \prime}$ :

$$
\begin{aligned}
\operatorname{ker} \mathcal{B}_{k}^{\prime} & =\left\{s_{k}, t_{k}\right\}, \\
\mathcal{B}_{k}^{\prime \prime} & =\mathcal{D}_{V_{k}}^{(k)} .
\end{aligned}
$$

By Lemma 3.3 we have $\mathcal{B}_{k}^{\prime \prime} \neq \mathcal{P}(X)$. The family $\mathfrak{B}=\left\{\mathcal{B}_{k}^{\prime}, \mathcal{B}_{k}^{\prime \prime}\right\}_{k \in \mathbb{N}^{+}}$is the $\sigma$-extension of the family $\left\{\mathcal{C}^{(k)}, \mathcal{D}^{(k)}\right\}_{k \in \mathbb{N}^{+}}$. It is clear that $\mathfrak{B}$ has no cycle (see the end of the proof of Theorem 2.17). 
3.8. Remark. For the proof of the sufficient condition of Theorem 1.17 we can use the following result:

Let $\mathfrak{A}=\left\{\mathcal{A}_{k}\right\}_{k \in \mathbb{N}^{+}}$be a family of $\sigma$-algebras and $\bigcup_{k=1}^{\infty} \mathcal{A}_{k} \neq \mathcal{P}(X)$. Then there exists $\sigma$-extension $\mathfrak{B}$ of $\mathfrak{A}$ such that if $\mathfrak{B}$ has a cycle then this cycle does not contain $\omega$-saturated algebras.

This result is a weaker result than Theorem 1.15 and for the proof of this result we do not use the Gitik-Shelah theorem. If $2^{\aleph_{0}}=\aleph_{1}$, then for the proof of the necessary condition of Theorem 1.17 we can use the simple Alouglu-Erdös theorem $[E]$ instead of the complicated Gitik-Shelah theorem. The Alouglu-Erdös theorem is the Gitik-Shelah theorem with the assumption that $\#(X)=\aleph_{1}$ (see Section 2.8).

\section{References}

[E] P. Erdös, Some remarks on set theory, Proceedings American Mathematical Society, vol. 1 (1950), 127-141.

[G-S] M. Gitik and S. Shelah, Forcing with ideals and simple forcing notions, Israel Journal of Mathematics, vol. 68 (1989), 129-160.

[Gl] A.M. Gleason, Projective topological spaces, Illinois Journal of Mathematics, vol. 2 (1958), 482-489.

[Gr1] L.Š. Grinblat, On sets not belonging to algebras of subsets, Memoirs of the American Mathematical Society, vol. 480 (1992).

[Gr2] L.Š. Grinblat, Algebras of sets and combinatorics, Translations of Mathematical Monographs, vol. 214, American Mathematical Society, Providence, RI, 2002.

[Gr3] L.Š. Grinblat, Theorems on sets not belonging to algebras, Electronic Research Announcements of the American Mathematical Society, vol. 10 (2004), 51-57.

[Gr4] L.S. Grinblat, On sets not belonging to algebras, The Journal of Symbolic Logic, vol. 72 (2007), 483-500.

[Gr5] L.S. Grinblat, Theorems with uniform conditions on sets not belonging to algebras, Topology Proceedings, vol. 33 (2009), 361-380.

[S] S. Shelah, Iterated forcing and normal ideals on $\omega_{1}$, Israel Journal of Mathematics, vol. 60(1987), 345-380.

[W] W.H. Woodin, The axiom of determinacy, forcing axioms, and the nonstationary ideal, de Gruyter, Berlin, 1999.

Department of Mathematics, Ariel University Center of Samaria, P.O. Box 3, Ariel 40700, ISRAEL

E-mail address: grinblat@ariel.ac.il 